\title{
Nonuniform Exponential Trichotomy for Linear Discrete-Time Systems in Banach Spaces
}

\author{
Xiao-qiu Song, Tian Yue, and Dong-qing Li \\ College of Sciences, China University of Mining and Technology, Xuzhou, Jiangsu 221008, China \\ Correspondence should be addressed to Xiao-qiu Song; sxqcumt@163.com
}

Received 24 June 2013; Revised 15 September 2013; Accepted 20 September 2013

Academic Editor: Gerassimos Barbatis

Copyright (C) 2013 Xiao-qiu Song et al. This is an open access article distributed under the Creative Commons Attribution License, which permits unrestricted use, distribution, and reproduction in any medium, provided the original work is properly cited.

\begin{abstract}
The aim of this paper is to give several characterizations for nonuniform exponential trichotomy properties of linear difference equations in Banach spaces. Well-known results for exponential stability and exponential dichotomy are extended to the case of nonuniform exponential trichotomy.
\end{abstract}

\section{Introduction}

In the mathematical literature of the last decades, the asymptotic properties of solutions of evolution equations in finite or infinite dimensional space have proved to be research area of large intensity. There were defined and developed concepts of the asymptotic behaviors, as stability, expansivity, dichotomy, and trichotomy (see [1-25] and the references therein), based on the fact that the dynamical systems which describe processes from economics, physical sciences, or engineering are extremely complex and the identification of the proper mathematical model is difficult.

As a natural generalization of exponential dichotomy, exponential trichotomy is one of the most complex asymptotic properties of dynamical systems arising from the central manifold theory. When people analyze the asymptotic behavior of dynamical systems, exponential trichotomy is a powerful tool. Starting from the idea that the center manifold of an equilibrium point of a dynamical system consists of orbits whose behavior around the equilibrium point is not controlled by either the attraction of the stable manifold or the repulsion of the unstable manifold, it is obvious that the concept of exponential dichotomy describes a rather idealistic situation when the solution is either exponentially stable on the stable subspaces or exponentially unstable on the unstable subspaces (see $[4,7,8,16]$ ). Thus, with motivation from the properties arising in bifurcation theory, a new asymptotic concept called exponential trichotomy which reflects a deeper analysis of the behavior of solutions of dynamical systems is introduced. Under this case the main idea in the study of the asymptotic behavior is to obtain, at any moment, a decomposition of the state space in three subspaces: a stable one, an instable one, and a third one, the central manifold.

The conception of trichotomy firstly arose in the works of Sacker and Sell [19] in 1976. They described trichotomy for linear differential systems by linear skew-product flows. Later, Elaydi and Hájek [6,7] gave the notions of exponential trichotomy for differential systems and for nonlinear differential systems, respectively. The case of difference equations received a special attention in the paper of Elaydi and Janglajew [8] where the authors deduced the first inputoutput criteria for exponential trichotomy, on one hand, and they introduced the first nonlinear discrete concepts of exponential trichotomy, on the other hand. Despite the increasing interest on this topic, most of papers were devoted to problems regarding the robustness of the exponential trichotomy (see $[1,9,10,12,15,25])$ and only in the past few years the existence criteria started to be obtained (see $[2,13,18,20-$ $22])$. For instance, in $[9,10]$ Hong and his partners studied the relationship between exponential trichotomy and the ergodic solutions of linear differential and difference equations with ergodic perturbations. In [20], the connections between the existence of exponential trichotomy of variational difference equations and the solvability of the associated variational control system were established. And in [22] B. Sasu and A.L. 
Sasu obtained some nonlinear conditions for the existence of the exponential trichotomy of skew-product flows in infinite dimensional spaces.

In this paper, we introduce the concept of nonuniform exponential trichotomy for linear difference equations which is an extension of classical concept of uniform exponential trichotomy. Our main objective is to give some characterizations for nonuniform exponential trichotomy properties of linear difference equations in Banach spaces, and variants for nonuniform exponential trichotomy of some well-known results in uniform exponential stability theory (Datko [5], Przyłuski and Rolewicz [17]) and exponential dichotomy theory (Popa et al. [16]) are obtained.

\section{Preliminaries}

Let $X$ be a real or complex Banach space. The norm on $X$ and on $\mathscr{B}(X)$ the Banach algebra of all bounded linear operators acting on $X$ will be denoted by $\|\cdot\|$. We denote $\Delta=\{(m, n) \in$ $\left.\mathbb{N}^{2}, m \geq n\right\}$ and $T=\left\{(m, p, n) \in \mathbb{N}^{3}, m \geq p \geq n\right\}$. Let $\mathscr{F}$ be the set of all nondecreasing functions $F: \mathbb{R}_{+} \rightarrow \mathbb{R}_{+}$with the properties $F(0)=0$ and $F(t)>0$ for every $t>0$. Let $I$ be the identity operator on $X$.

In the present paper we consider linear discrete-time system of difference equations

$$
x_{n+1}=A(n) x_{n}, \quad n \in \mathbb{N},
$$

where $A: \mathbb{N} \rightarrow \mathscr{B}(X)$ is a sequence in $\mathscr{B}(X)$. Then every solution $x=\left\{x_{n}\right\}$ of the system (1) is given by

$$
x_{m}=U(m, n) x_{n}
$$

for all $(m, n) \in \Delta$, where the mapping $U: \Delta \rightarrow \mathscr{B}(X)$ is defined by

$$
U(m, n):= \begin{cases}I, & m=n \\ A(m-1) \cdots A(n), & m>n .\end{cases}
$$

It is easy to see that $U(m, p) U(p, n)=U(m, n)$, for all $(m$, $p, n) \in T$.

For the particular case when (1) is autonomous, that is, $A(n)=A \in \mathscr{B}(X)$ for all $n \in \mathbb{N}$, then $U(m, n)=A^{m-n}$ for all $(m, n) \in \Delta$.

Definition 1. An application $P: \mathbb{N} \rightarrow \mathscr{B}(X)$ is said to be a projection family on $X$ if

$$
P^{2}(n)=P(n),
$$

for all $n \in \mathbb{N}$.

Definition 2. Three projection families $P_{1}, P_{2}, P_{3}: \mathbb{N} \rightarrow$ $\mathscr{B}(X)$ are said to be compatible with the system (1), if

(c1) $P_{1}(n)+P_{2}(n)+P_{3}(n)=I$, for all $n \in \mathbb{N}$,

(c2) $P_{i}(n) P_{j}(n)=0$, for all $n \in \mathbb{N}$, for all $i, j \in$ $\{1,2,3\}, i \neq j$,

(c3) $\left\|P_{i}(n) x+P_{j}(n) x\right\|^{2}=\left\|P_{i}(n) x\right\|^{2}+\left\|P_{j}(n) x\right\|^{2}$, for all $n \in \mathbb{N}$, all $x \in X$, and all $i, j \in\{1,2,3\}, i \neq j$,

(c4) $A(n) P_{i}(n)=P_{i}(n+1) A(n)$, for all $n \in \mathbb{N}$ and all $i \in$ $\{1,2,3\}$.
Remark 3. If $P_{1}, P_{2}, P_{3}: \mathbb{N} \rightarrow \mathscr{B}(X)$ are projection families compatible with the system (1) then

$$
U(m, n) P_{i}(n)=P_{i}(m) U(m, n)
$$

for all $i \in\{1,2,3\}$ and all $(m, n) \in \Delta$.

Definition 4. The linear discrete-time system (1) is said to be uniformly exponentially trichotomic if there exist three projection families $\left\{P_{i}\right\}_{i \in\{1,2,3\}}$ compatible with the system (1), constants $v_{1}, v_{2}, v_{3}$, and $v_{4}$ with the property $v_{1} \leq v_{2}<0<$ $v_{3} \leq v_{4}$, and $N \geq 1$ such that

$$
\begin{aligned}
& \left(\text { uet }_{1}\right)\left\|U(m, n) P_{1}(n) x\right\| \leq N\left\|P_{1}(n) x\right\| e^{v_{1}(m-n)}, \\
& \left(\text { uet }_{2}\right)\left\|P_{2}(n) x\right\| \leq N\left\|U(m, n) P_{2}(n) x\right\| e^{-v_{4}(m-n)}, \\
& \left(\text { uet }_{3}\right)\left\|U(m, n) P_{3}(n) x\right\| \leq N\left\|P_{3}(n) x\right\| e^{v_{3}(m-n)}, \\
& \left(\text { uet }_{4}\right)\left\|P_{3}(n) x\right\| \leq N\left\|U(m, n) P_{3}(n) x\right\| e^{-v_{2}(m-n)},
\end{aligned}
$$

for all $(m, n, x) \in \Delta \times X$.

Example 5. Let $X=\mathbb{R}^{3}$ with the Euclidean norm. The application $\mathrm{A}: \mathbb{N} \rightarrow \mathscr{B}\left(\mathbb{R}^{3}\right)$ is defined by

$$
\begin{array}{r}
A(n)\left(x_{1}, x_{2}, x_{3}\right)=\left(a e^{\lambda_{n}} x_{1}, \frac{x_{2}}{a}, \frac{x_{3}}{e}\right), \\
\forall\left(n, x_{1}, x_{2}, x_{3}\right) \in \mathbb{N} \times \mathbb{R}^{3},
\end{array}
$$

where $a \in\left(0, e^{-3}\right)$ and $\lambda_{n}=(n+2) \cos \ln (n+2)-(n+$ 1) $\cos \ln (n+1)$.

Let us consider the projection families $P_{1}, P_{2}, P_{3}: \mathbb{N} \rightarrow$ $\mathscr{B}\left(\mathbb{R}^{3}\right)$ defined by

$$
\begin{aligned}
& P_{1}(n)\left(x_{1}, x_{2}, x_{3}\right)=\left(x_{1}, 0,0\right), \\
& P_{2}(n)\left(x_{1}, x_{2}, x_{3}\right)=\left(0, x_{2}, 0\right), \\
& P_{3}(n)\left(x_{1}, x_{2}, x_{3}\right)=\left(0,0, x_{3}\right)
\end{aligned}
$$

for all $n \in \mathbb{N}$ and all $x=\left(x_{1}, x_{2}, x_{3}\right) \in X$.

A simple calculus shows that

$$
\begin{aligned}
& U(m, n) P_{1}(n) x=\left(a^{m-n} e^{\mu_{m n}} x_{1}, 0,0\right), \\
& U(m, n) P_{2}(n) x=\left(0, a^{-(m-n)} x_{2}, 0\right), \\
& U(m, n) P_{3}(n) x=\left(0,0, e^{-(m-n)} x_{3}\right),
\end{aligned}
$$

where $\mu_{m n}=(m+1) \cos \ln (m+1)-(n+1) \cos \ln (n+1)$.

If we denote $(x)=x \cos \ln x$, then by Lagrange's mean value theorem it results that for every $n \in \mathbb{N}$ there exists $\tau_{n} \in(n+1, n+2)$ such that

$$
\lambda_{n}=\varphi(n+2)-\varphi(n+1)=\cos \ln \tau_{n}-\sin \ln \tau_{n}
$$

and hence $\left|\lambda_{n}\right| \leq \sqrt{2} \leq 2$ for every $n \in \mathbb{N}$.

This implies that

$$
\sup _{n \in \mathbb{N}}\|A(n)\| \leq a e^{2}+\frac{1}{a}+\frac{1}{e}, \quad\left|\mu_{m n}\right| \leq 2(m-n) \text {. }
$$


In the following we obtain the relations

$$
\begin{aligned}
e^{m-n}\left\|U(m, n) P_{1}(n) x\right\| & \leq e^{\varepsilon(m-n)+\mu_{m n}} a^{m-n}\left|x_{1}\right| \\
& \leq\left|x_{1}\right|=\left\|P_{1}(n) x\right\|, \\
e^{m-n}\left\|P_{2}(n) x\right\| & \leq e^{3(m-n)}\left|x_{2}\right| \leq a^{-(m-n)}\left|x_{2}\right| \\
& =\left\|U(m, n) P_{2}(n) x\right\|, \\
\left\|U(m, n) P_{3}(n) x\right\| & =e^{-(m-n)}\left|x_{3}\right| \\
& \leq e^{(m-n)}\left|x_{3}\right|=\left\|P_{3}(n) x\right\| e^{(m-n)}, \\
\left\|P_{3}(n) x\right\| & =\left|x_{3}\right| \leq\left\|U(m, n) P_{3}(n) x\right\| e^{(m-n)},
\end{aligned}
$$

for all $(m, n, x) \in \Delta \times \mathbb{R}^{3}$, where $\varepsilon=\ln \left(a e^{2}\right)^{-1}>\ln e=1$. Thus Definition 4 is satisfied for $N=-v_{1}=-v_{2}=v_{3}=v_{4}=1$; hence, the system (1) is uniformly exponentially trichotomic.

Definition 6. The linear discrete-time system (1) is said to be nonuniformly exponentially trichotomic if there exist a nondecreasing sequence of real numbers $N: \mathbb{N} \rightarrow \mathbb{R}_{+}^{*}$, constants $v_{1}, v_{2}, v_{3}$, and $v_{4}$ with the property $v_{1} \leq v_{2}<0<$ $v_{3} \leq v_{4}$, and three projection families $\left\{P_{i}\right\}_{i \in\{1,2,3\}}$ compatible with the system (1) such that

$$
\begin{aligned}
& \left(\text { net }_{1}\right)\left\|U(m, n) P_{1}(n) x\right\| \leq N(n)\left\|P_{1}(n) x\right\| e^{v_{1}(m-n)}, \\
& \left(\text { net }_{2}\right)\left\|P_{2}(n) x\right\| \leq N(m)\left\|U(m, n) P_{2}(n) x\right\| e^{-v_{4}(m-n)}, \\
& \left(\text { net }_{3}\right)\left\|U(m, n) P_{3}(n) x\right\| \leq N(n)\left\|P_{3}(n) x\right\| e^{v_{3}(m-n)}, \\
& \left(\text { net }_{4}\right)\left\|P_{3}(n) x\right\| \leq N(m)\left\|U(m, n) P_{3}(n) x\right\| e^{-v_{2}(m-n)},
\end{aligned}
$$

for all $(m, n, x) \in \Delta \times X$.

Remark 7. Consider the following.

(i) For $P_{3}=0$ in Definition 6 we obtain the property of nonuniform exponential dichotomy.

(ii) For $P_{2}=P_{3}=0$, the property of nonuniform exponential stability is obtained. It follows that a nonuniformly exponentially stable linear discrete-time system is nonuniformly exponentially dichotomic and, further, nonuniformly exponentially trichotomic.

(iii) For $P_{1}=P_{3}=0$, we obtain the property of nonuniform exponential expansivity. Also it is easy to see that the property of nonuniform exponential expansivity implies the nonuniform exponential dichotomy and, further, the nonuniform exponential trichotomy.

Remark 8. The linear discrete-time system (1) is nonuniformly exponentially trichotomic if and only if there exist a nondecreasing sequence of real numbers $N: \mathbb{N} \rightarrow \mathbb{R}^{*}$, constants $v_{1}, v_{2}, v_{3}$, and $v_{4}$ with the property $v_{1} \leq v_{2}<0<$ $v_{3} \leq v_{4}$, and three projection families $\left\{P_{i}\right\}_{i \in\{1,2,3\}}$ compatible with the system (1) such that

(a)

$$
\left\|U(m, n) P_{1}(n) x\right\| \leq N(p)\left\|U(p, n) P_{1}(n) x\right\| e^{v_{1}(m-p)},
$$

(b)

$$
\left\|U(p, n) P_{2}(n) x\right\| \leq N(m)\left\|U(m, n) P_{2}(n) x\right\| e^{-v_{4}(m-p)},
$$

(c)

$\left\|U(m, n) P_{3}(n) x\right\| \leq N(p)\left\|U(p, n) P_{3}(n) x\right\| e^{v_{3}(m-p)}$,

$$
\left\|U(p, n) P_{3}(n) x\right\| \leq N(m)\left\|U(m, n) P_{3}(n) x\right\| e^{-v_{2}(m-p)},
$$

for all $(m, p, n, x) \in T \times X$.

Remark 9. It is obvious that if the system (1) is uniformly exponentially trichotomic then it is nonuniformly exponentially trichotomic. But the converse statement is not necessarily valid. This fact is illustrated by the following example.

Example 10. Let $\mathrm{X}=\mathbb{R}^{3}$ with the Euclidean norm. The application $A: \mathbb{N} \rightarrow \mathscr{B}\left(\mathbb{R}^{3}\right)$ is defined by

$$
\begin{array}{r}
A(n)\left(x_{1}, x_{2}, x_{3}\right)=\left(a x_{1}, x_{2}, x_{3}\right), \\
\forall\left(n, x_{1}, x_{2}, x_{3}\right) \in \mathbb{N} \times \mathbb{R}^{3},
\end{array}
$$

where $a \in\left(0, e^{-1}\right]$. We consider the projection families $P_{1}, P_{2}, P_{3}: \mathbb{N} \rightarrow \mathscr{B}\left(\mathbb{R}^{3}\right)$,

$$
\begin{gathered}
P_{1}(n)\left(x_{1}, x_{2}, x_{3}\right)=\left(x_{1}, 0,0\right), \\
P_{2}(n)\left(x_{1}, x_{2}, x_{3}\right)=\left(0, x_{2}, 0\right), \\
P_{3}(n)\left(x_{1}, x_{2}, x_{3}\right)=\left(0,0, x_{3}\right)
\end{gathered}
$$

for all $n \in \mathbb{N}$ and all $x=\left(x_{1}, x_{2}, x_{3}\right) \in X$, compatible with the system (1).

Then

$$
\begin{gathered}
U(m, n) P_{1}(n) x=\left(a^{m-n} x_{1}, 0,0\right), \\
U(m, n) P_{2}(n) x=\left(0, x_{2}, 0\right), \\
U(m, n) P_{3}(n) x=\left(0,0, x_{3}\right) .
\end{gathered}
$$

Further we have that

$$
\begin{aligned}
e^{m-n}\left\|U(m, n) P_{1}(n) x\right\| & =(e a)^{m-n}\left|x_{1}\right| \\
& \leq\left|x_{1}\right| \leq e^{n}\left|x_{1}\right| \\
& =N(n)\left\|P_{1}(n) x\right\|, \\
e^{m-n}\left\|P_{2}(n) x\right\| & =e^{m-n}\left|x_{2}\right| \leq e^{m}\left|x_{2}\right| \\
& =N(m)\left\|U(m, n) P_{2}(n) x\right\|, \\
\frac{1}{N(m)} e^{-(m-n)}\left\|P_{3}(n) x\right\| & =e^{-m} \cdot e^{-(m-n)}\left|x_{3}\right| \\
& \leq\left\|U(m, n) P_{3}(n) x\right\| \\
& =\left|x_{3}\right| \leq e^{n} \cdot e^{m-n}\left\|P_{3}(n) x\right\|
\end{aligned}
$$


for all $(m, n, x) \in \Delta \times \mathbb{R}^{3}$. Thus Definition 6 is satisfied for $N(n)=e^{n}$ and $v_{1}=v_{2}=-v_{3}=-v_{4}=-1$; hence, the system (1) is nonuniformly exponentially trichotomic.

On the other hand, if we suppose that system (1) is uniformly exponentially trichotomic, then there exist two constants $N \geq 1$ and $v_{4}>0$ such that

$$
\begin{aligned}
\left|x_{2}\right| & =\left\|P_{2}(n) x\right\| \leq N\left\|U(m, n) P_{2}(n) x\right\| e^{-v_{4}(m-n)} \\
& =N\left|x_{2}\right| e^{-v_{4}(m-n)}
\end{aligned}
$$

for all $(m, n, x) \in \Delta \times \mathbb{R}^{3}$. This implies that $e^{v_{4}(m-n)} \leq N$ for all $(m, n) \in \Delta$, which is false.

\section{The Main Results}

Theorem 11. The linear discrete-time system (1) is nonuniformly exponentially trichotomic if and only if there exist a function $F \in \mathscr{F}$ and three projection families $\left\{P_{i}\right\}_{i \in\{1,2,3\}}$ compatible with the system (1) such that the following relations hold:

(i) There exist a constant $d_{1}>0$ and a sequence of positive real numbers $\left\{\xi_{n}\right\}_{n \in \mathbb{N}}$ such that

$$
\sum_{k=n}^{m} F\left(e^{d_{1}(k-n)}\left\|U(k, n) P_{1}(n) x\right\|\right) \leq F\left(\xi_{n}\left\|P_{1}(n) x\right\|\right),
$$

(ii) There exist a constant $d_{2}>0$ and a sequence of positive real numbers $\left\{\eta_{n}\right\}_{n \in \mathbb{N}}$ such that

$$
\begin{aligned}
& \sum_{k=n}^{m} F\left(e^{-d_{2}(k-n)}\left\|U(k, n) P_{2}(n) x\right\|\right) \\
& \quad \leq F\left(\eta_{m} e^{-d_{2}(m-n)}\left\|U(m, n) P_{2}(n) x\right\|\right),
\end{aligned}
$$

(iii) There exist a constant $d_{3}>0$ and a sequence of positive real numbers $\left\{\alpha_{n}\right\}_{n \in \mathbb{N}}$ such that

$$
\begin{aligned}
& \sum_{k=p}^{m} F\left(e^{-d_{3}(k-n)}\left\|U(k, n) P_{3}(n) x\right\|\right) \\
& \quad \leq F\left(\alpha_{n} e^{-d_{3}(p-n)}\left\|U(p, n) P_{3}(n) x\right\|\right),
\end{aligned}
$$

(iv) There exist a constant $d_{4}>0$ and a sequence of positive real numbers $\left\{\beta_{n}\right\}_{n \in \mathbb{N}}$ such that

$$
\begin{aligned}
& \sum_{k=p}^{m} F\left(e^{d_{4}(k-n)}\left\|U(k, n) P_{3}(n) x\right\|\right) \\
& \quad \leq F\left(\beta_{m} e^{d_{4}(m-n)}\left\|U(m, n) P_{3}(n) x\right\|\right)
\end{aligned}
$$

for all $(m, p, n, x) \in T \times X$.

Proof. (a) $\Leftrightarrow$ (i) Necessity. We consider $F(t)=t, t \geq 0$. As system (1) is nonuniformly exponentially trichotomic, Remark 8 assures the existence of a constant $v_{1}<0$, a sequence of real numbers $N: \mathbb{N} \rightarrow \mathbb{R}_{+}^{*}$, and a projection family
$P_{1}$ such that (a) holds. We obtain for $d_{1}=-\left(v_{1} / 2\right)>0$ and according to (a)

$$
\begin{aligned}
& \sum_{k=n}^{m} e^{d_{1}(k-n)}\left\|U(k, n) P_{1}(n) x\right\| \\
& \leq N(n) \sum_{k=n}^{m} e^{d_{1}(k-n)} e^{v_{1}(k-n)}\left\|U(n, n) P_{1}(n) x\right\| \\
& =N(n)\left\|P_{1}(n) x\right\| \sum_{k=n}^{m} e^{\left(v_{1} / 2\right)(k-n)} \\
& \leq \xi_{n}\left\|P_{1}(n) x\right\|, \quad \forall(m, n, x) \in \Delta \times X,
\end{aligned}
$$

where we have denoted $\xi_{n}=\left(1 /\left(1-e^{-d_{1}}\right)\right) N(n), n \in \mathbb{N}$.

Sufficiency. According to the hypothesis, if we consider $k=m$ then

$$
F\left(e^{d_{1}(m-n)}\left\|U(m, n) P_{1}(n) x\right\|\right) \leq F\left(\xi_{n}\left\|P_{1}(n) x\right\|\right)
$$

for all $(m, n, x) \in \Delta \times X$. By means of the properties of function $F$, we have

$$
\left\|U(m, n) P_{1}(n) x\right\| \leq \xi_{n}\left\|P_{1}(n) x\right\| e^{-d_{1}(m-n)} .
$$

Thus relation (a) is obtained.

Similarly the other equivalences can also be proved, that is, (b) $\Leftrightarrow$ (ii), (c) $\Leftrightarrow$ (iii), and (d) $\Leftrightarrow$ (iv).

Remark 12. The preceding theorem is an extension for the case of nonuniform exponential trichotomy of a result due to Popa et al. in [16]. Additionally, it is variant for the case of nonuniform exponential trichotomy property of a well-known theorem due to Przyłuski and Rolewicz [17] for exponential stability. If we consider $F(t)=t^{p}, t \geq 0, p>0$, then Theorem 11 can be considered a version for the case of nonuniform exponential trichotomy of some results due to Datko [5].

It is well known that the exponential dichotomy involves two commuting projection families. In order to emphasize the natural extension of the nonuniform exponential trichotomy relative to the property of dichotomy, we will present a characterization by means of two commuting projection families, introduced by the following.

Definition 13. Two projection families $R_{1}, R_{2}: \mathbb{N} \rightarrow \mathscr{B}(X)$ are said to be compatible with the system (1), if

(r1) $R_{i}(n) R_{j}(n)=0$, for all $n \in \mathbb{N}$, for all $i, j \in\{1,2\}$, $i \neq j$,

(r2) $\left\|R_{1}(n) x+R_{2}(n) x\right\|^{2}=\left\|R_{1}(n) x\right\|^{2}+\left\|R_{2}(n) x\right\|^{2}$, for all $n \in \mathbb{N}$, all $x \in X$,

(r3) $\left\|\left[I-R_{1}(n)\right]\right\|^{2}=\left\|\left[I-R_{1}(n)-R_{2}(n)\right] x\right\|^{2}+$ $\left\|R_{2}(n) x\right\|^{2}$, for all $n \in \mathbb{N}$, all $x \in X$,

(r4) $\left\|\left[I-R_{2}(n)\right]\right\|^{2}=\left\|\left[I-R_{1}(n)-R_{2}(n)\right] x\right\|^{2}+$ $\left\|R_{1}(n) x\right\|^{2}$, for all $n \in \mathbb{N}$, all $x \in X$,

(r5) $A(n) R_{i}(n)=R_{i}(n+1) A(n)$, for all $n \in \mathbb{N}$ and all $i \in\{1,2\}$. 
Theorem 14. The linear discrete-time system (1) is nonuniformly exponentially trichotomic if and only if there exist a nondecreasing sequence of real numbers $N_{1}: \mathbb{N} \rightarrow \mathbb{R}_{+}^{*}$, constants $\mu>v>0$, and two projection families $\left\{R_{i}\right\}_{i \in\{1,2\}}$ compatible with the system (1) such that

$\left(a^{\prime}\right)$

$$
\left\|U(m, n) R_{1}(n) x\right\| \leq N_{1}(p)\left\|U(p, n) R_{1}(n) x\right\| e^{-\mu(m-p)},
$$

$\left(b^{\prime}\right)$

$$
\left\|U(p, n) R_{2}(n) x\right\| \leq N_{1}(m)\left\|U(m, n) R_{2}(n) x\right\| e^{-v(m-p)},
$$

$\left(c^{\prime}\right)$

$$
\begin{aligned}
& \left\|U(m, n)\left[I-R_{2}(n)\right] x\right\| \\
& \quad \leq N_{1}(p)\left\|U(p, n)\left[I-R_{2}(n)\right] x\right\| e^{v(m-p)},
\end{aligned}
$$

$\left(d^{\prime}\right)$

$$
\begin{aligned}
& \left\|U(p, n)\left[I-R_{1}(n)\right] x\right\| \\
& \quad \leq N_{1}(m)\left\|U(m, n)\left[I-R_{1}(n)\right] x\right\| e^{\mu(m-p)},
\end{aligned}
$$

for all $(m, p, n, x) \in T \times X$.

Proof. Necessity. As the system (1) is nonuniformly exponentially trichotomic, according to Remark 8 there exist three projection families $\left\{P_{i}\right\}_{i \in\{1,2,3\}}$ compatible with the system (1), constants $v_{1} \leq v_{2}<0<v_{3} \leq v_{4}$, and a nondecreasing sequence of real numbers $N: \mathbb{N} \rightarrow \mathbb{R}^{*}$ such that relations (a)-(d) hold.

If we denote $R_{1}=P_{1}$ and $R_{2}=P_{2}$ then conditions (rl)-(r5) of Definition 13 result from (c1)-(c4) of Definition 2. Thus, the projection families $R_{1}$ and $R_{2}$ are compatible with the system (1).

Let us define $\mu=-v_{1}=-v_{2}>0, v=v_{3}=v_{4}>0$, and $N_{1}(n)=N(n), n \in \mathbb{N}$. It is obvious that relations $\left(a^{\prime}\right)$ and $\left(b^{\prime}\right)$ hold. Using conditions (c3), (c4), (b), and (d) we obtain

$$
\begin{aligned}
&\left\|U(p, n)\left[I-R_{1}(n)\right] x\right\|^{2} \\
&=\left\|U(p, n)\left[P_{2}(n)+P_{3}(n)\right] x\right\|^{2} \\
&=\left\|U(p, n) P_{2}(n) x\right\|^{2}+\left\|U(p, n) P_{3}(n) x\right\|^{2} \\
& \leq N(m)^{2}\left\|U(m, n) P_{2}(n) x\right\|^{2} e^{-2 v_{4}(m-p)} \\
&+N(m)^{2}\left\|U(m, n) P_{3}(n) x\right\|^{2} e^{-2 v_{2}(m-p)} \\
& \leq N(m)^{2}\left\|U(m, n)\left[P_{2}(n)+P_{3}(n)\right] x\right\|^{2} e^{-2 v_{2}(m-p)} \\
&= N_{1}(m)^{2}\left\|U(m, n)\left[I-R_{1}(n)\right] x\right\|^{2} e^{2 \mu(m-p)}
\end{aligned}
$$

for all $(m, p, n, x) \in T \times X$. Hence, relation $\left(d^{\prime}\right)$ holds.

The proof of $\left(c^{\prime}\right)$ is similar.
Sufficiency. We consider the projection families

$$
\begin{gathered}
P_{1}=R_{1}, \quad P_{2}=R_{2}, \\
P_{3}=I-R_{1}-R_{2}=\left(I-R_{1}\right)\left(I-R_{2}\right) .
\end{gathered}
$$

These are compatible with the system (1).

Let us define $v_{1}=v_{2}=-\mu<0, v_{3}=v_{4}=v>0$, and $N(n)=N_{1}(n), n \in \mathbb{N}$. It is clear that $\left(a^{\prime}\right) \Leftrightarrow(\mathrm{a})$ and $\left(b^{\prime}\right) \Leftrightarrow(\mathrm{b})$. By condition $\left(c^{\prime}\right)$ we obtain

$$
\begin{aligned}
\| U & (m, n) P_{3}(n) x \| \\
& =\left\|U(m, n)\left[I-R_{1}(n)\right]\left[I-R_{2}(n)\right] x\right\| \\
& \leq N_{1}(p)\left\|U(p, n)\left[I-R_{1}(n)\right]\left[I-R_{2}(n)\right] x\right\| e^{\nu(m-p)} \\
& =N(p)\left\|U(p, n) P_{3}(n) x\right\| e^{\nu_{3}(m-p)}
\end{aligned}
$$

for all ( $m, p, n, x) \in T \times X$, and hence (c) holds.

Similarly, we can prove that $\left(d^{\prime}\right)$ implies relation (d). Finally, by Remark 8 we conclude that the system (1) is nonuniformly exponentially trichotomic.

\section{Acknowledgments}

The authors would like to express their sincere thanks to the reviewer for the valuable suggestions and comments which have improved the quality of the paper. This work was supported by "the Fundamental Research Funds for the Central Universities" (no. 2013XK03).

\section{References}

[1] L. Barreira and C. Valls, "Robustness of nonuniform exponential trichotomies in Banach spaces," Journal of Mathematical Analysis and Applications, vol. 351, no. 1, pp. 373-381, 2009.

[2] L. Barreira and C. Valls, "Lyapunov sequences for exponential trichotomies," Nonlinear Analysis: Theory, Methods and Applications, vol. 72, no. 1, pp. 192-203, 2010.

[3] L. Barreira and C. Valls, "Center manifolds for infinite delay," Journal of Differential Equations, vol. 247, no. 4, pp. 1297-1310, 2009.

[4] W. A. Coppel, Dichotomies in Stability Theory, vol. 629 of Lecture Notes in Mathematics, Springer, Berlin, Germany, 1978.

[5] R. Datko, "Uniform asymptotic stability of evolutionary processes in a Banach space," SIAM Journal on Mathematical Analysis, vol. 3, pp. 428-445, 1972.

[6] S. Elaydi and O. Hajek, "Exponential trichotomy of differential systems," Journal of Mathematical Analysis and Applications, vol. 129, no. 2, pp. 362-374, 1988.

[7] S. Elaydi and O. Hájek, "Exponential dichotomy and trichotomy of nonlinear differential equations," Differential and Integral Equations, vol. 3, no. 6, pp. 1201-1224, 1990.

[8] S. Elaydi and K. Janglajew, "Dichotomy and trichotomy of difference equations," Journal of Difference Equations and Applications, vol. 3, pp. 417-448, 1998.

[9] J. Hong, R. Obaya, and A. S. Gil, "Exponential trichotomy and a class of ergodic solutions of differential equations with ergodic perturbations," Applied Mathematics Letters, vol. 12, no. 1, pp. 7-13, 1999. 
[10] J. Hong, R. Obaya, and A. Sanz, "Existence of a class of ergodic solutions implies exponential trichotomy," Applied Mathematics Letters, vol. 12, no. 4, pp. 43-45, 1999.

[11] J. López-Fenner and M. Pinto, "( $h, k)$-Trichotomies and asymptotics of nonautonomous difference systems," Computers and Mathematics with Applications, vol. 33, no. 10, pp. 105-124, 1997.

[12] S. Matucci, "The $l^{p}$ trichotomy for difference systems and applications," Archivum Mathematicum, vol. 36, no. 5, pp. 519$529,2000$.

[13] M. Megan and C. Stoica, "On uniform exponential trichotomy of evolution operators in Banach spaces," Integral Equations and Operator Theory, vol. 60, no. 4, pp. 499-506, 2008.

[14] M. Megan and C. Stoica, "Equivalent definitions for uniform exponential trichotomy of evolution operators in Banach spaces," in Hot Topics in Operator Theory, vol. 9 of Theta Series in Advanced Mathematics, pp. 151-158, 2008.

[15] G. Papaschinopoulos, "On exponential trichotomy of linear difference equations," Applicable Analysis, vol. 40, pp. 89-109, 1991.

[16] I.-L. Popa, M. Megan, and T. Ceausu, "Exponential dichotomies for linear discrete-time systems in Banach spaces," Applicable Analysis and Discrete Mathematics, vol. 6, pp. 140-155, 2012.

[17] K. M. Przyłuski and S. Rolewicz, "On stability of linear timevarying infinite-dimensional discrete-time systems," Systems \& Control Letters, vol. 4, no. 5, pp. 307-315, 1984.

[18] C. Stoica, "Pointwise trichotomy for skew-evolution semiflows on Banach spaces," Journal of Mathematical Sciences, vol. 161, no. 2, pp. 327-336, 2009.

[19] R. J. Sacker and G. R. Sell, "Existence of dichotomies and invariant splittings for linear differential systems, III," Journal of Differential Equations, vol. 22, no. 2, pp. 497-522, 1976.

[20] A. L. Sasu and B. Sasu, "Exponential trichotomy for variational difference equations," Journal of Difference Equations and Applications, vol. 15, no. 7, pp. 693-718, 2009.

[21] A. L. Sasu and B. Sasu, "Input-output admissibility and exponential trichotomy of difference equations," Journal of Mathematical Analysis and Applications, vol. 380, no. 1, pp. 17-32, 2011.

[22] B. Sasu and A. L. Sasu, "Nonlinear criteria for the existence of the exponential trichotomy in infinite dimensional spaces," Nonlinear Analysis: Theory, Methods and Applications, vol. 74, no. 15, pp. 5097-5110, 2011.

[23] B. Sasu, "New criteria for exponential expansiveness of variational difference equations," Journal of Mathematical Analysis and Applications, vol. 327, no. 1, pp. 287-297, 2007.

[24] B. Sasu, "On dichotomous behavior of variational difference equations and applications," Discrete Dynamics in Nature and Society, vol. 2009, Article ID 140369, 16 pages, 2009.

[25] C. Cuevas and C. Vidal, "Weighted exponential trichotomy of linear difference equations," Dynamics of Continuous, Discrete and Impulsive Systems Series A, vol. 15, no. 3, pp. 353-379, 2008. 


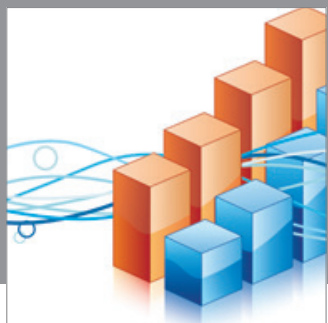

Advances in

Operations Research

mansans

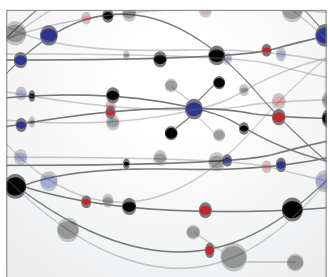

The Scientific World Journal
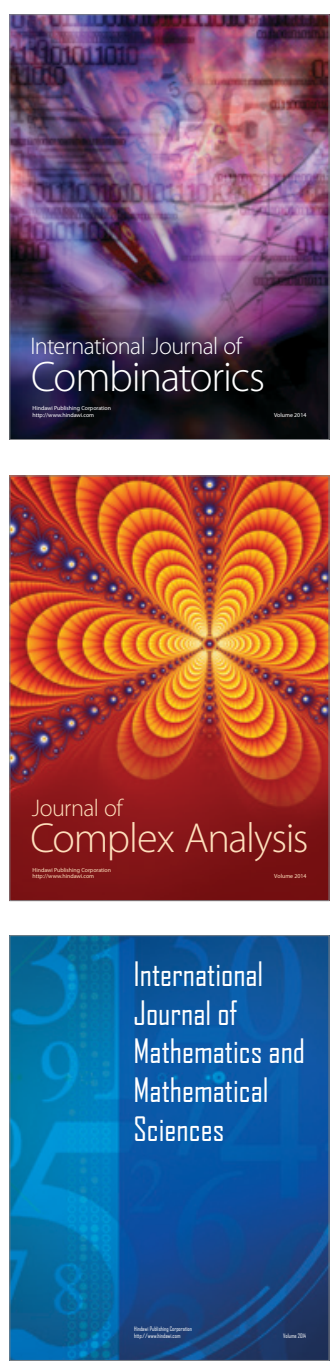
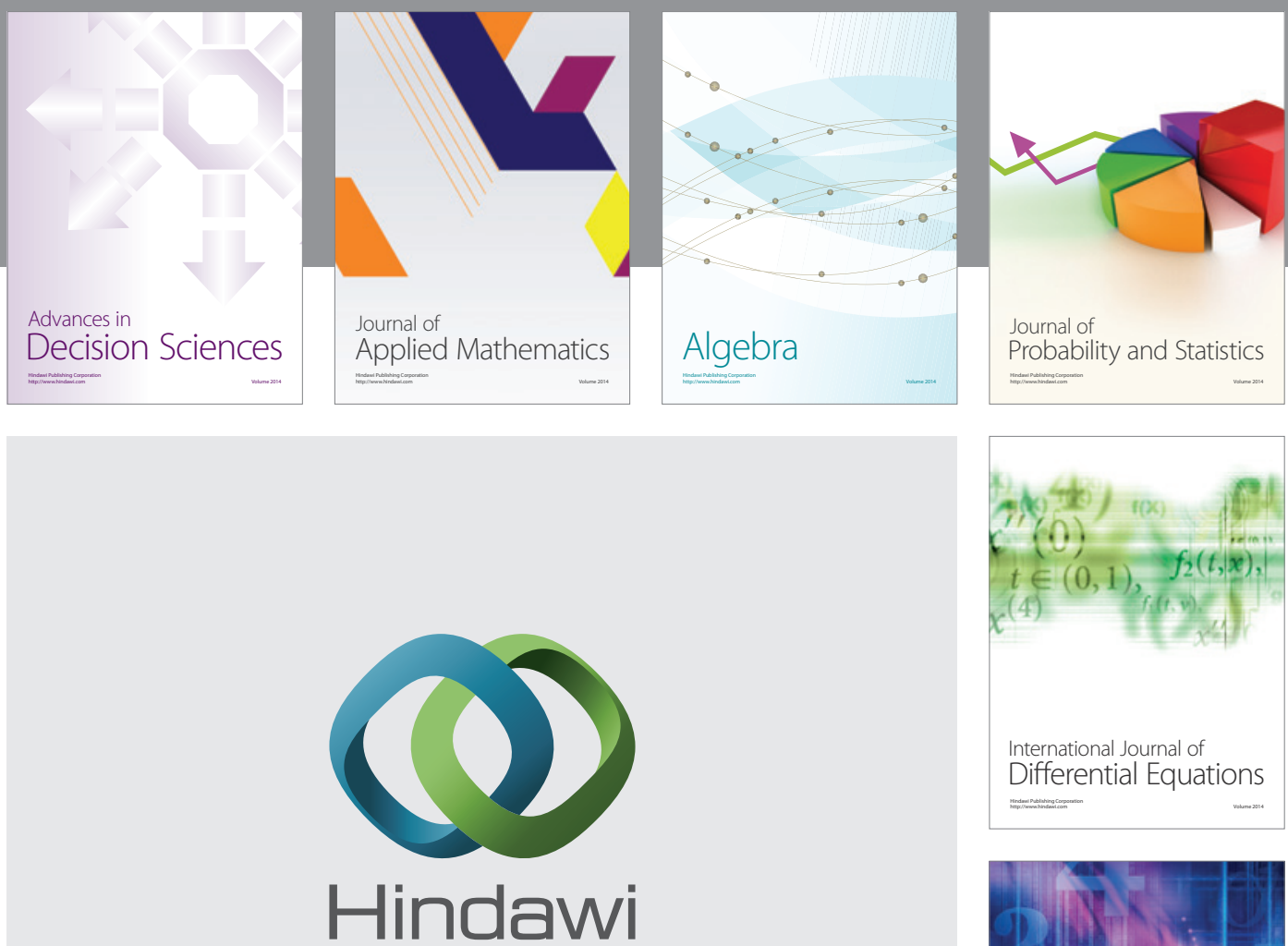

Submit your manuscripts at http://www.hindawi.com
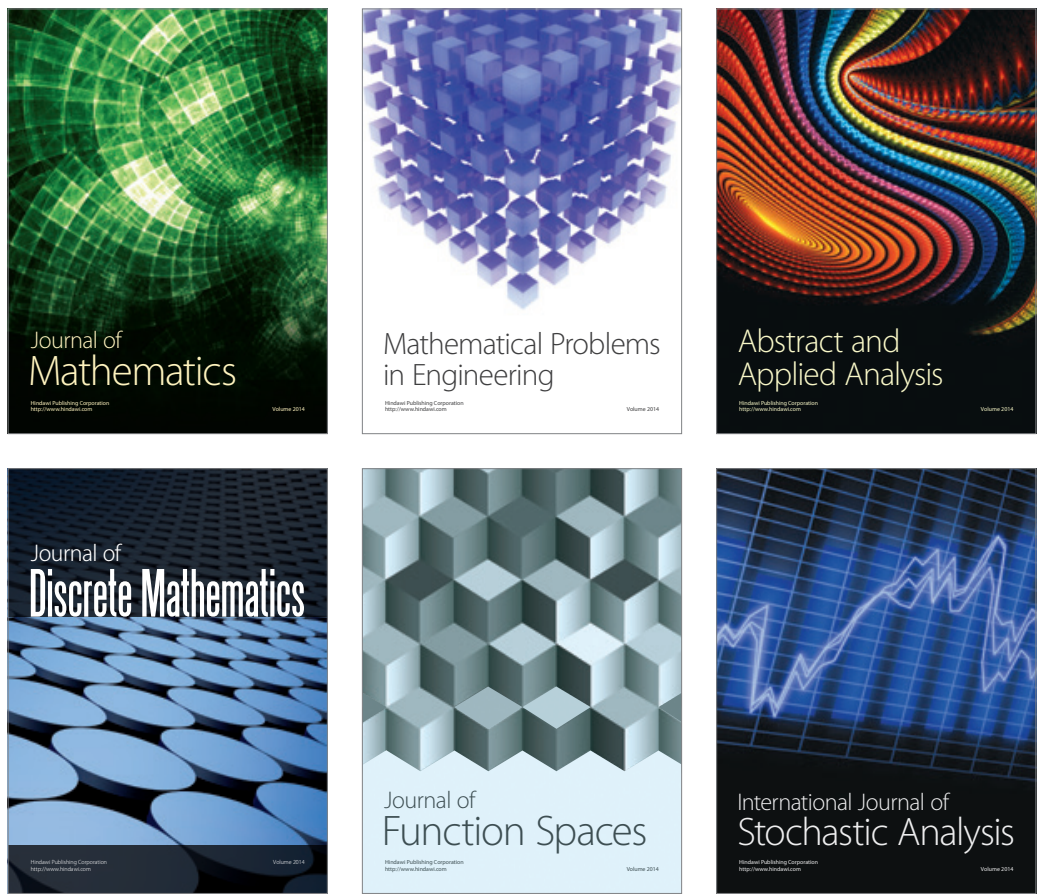

Journal of

Function Spaces

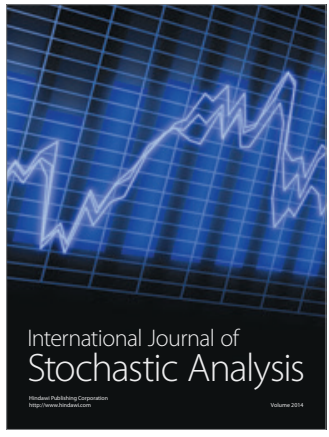

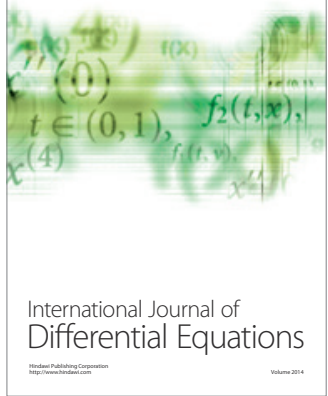
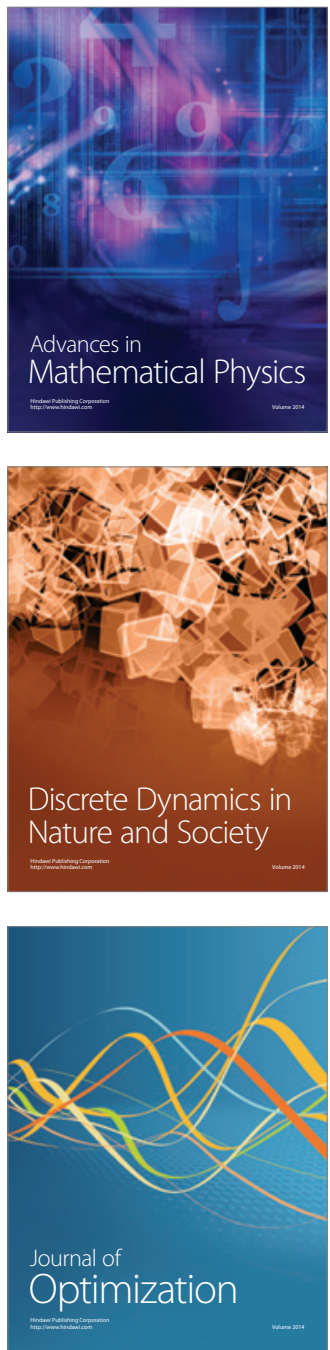